\title{
Wehrl entropy production rate across a dynamical quantum phase transition
}

\author{
B. O. Goes $\odot,{ }^{1}$ G. T. Landi, ${ }^{1}$ E. Solano, ${ }^{2,3,4,5}$ M. Sanz $\odot,{ }^{2,3,5}$ and L. C. Céleri ${ }^{6,2}$ \\ ${ }^{1}$ Instituto de Física, Universidade de São Paulo, CEP 05314-970, São Paulo, São Paulo, Brazil \\ ${ }^{2}$ Department of Physical Chemistry, University of the Basque Country UPV/EHU, Apartado 644, 48080 Bilbao, Spain \\ ${ }^{3}$ IKERBASQUE, Basque Foundation for Science, Maria Diaz de Haro 3, 48013 Bilbao, Spain \\ ${ }^{4}$ International Center of Quantum Artificial Intelligence for Science and Technology (QuArtist) \\ and Department of Physics, Shanghai University, 200444 Shanghai, China \\ ${ }^{5}$ IQM, Nymphenburgerstr. 86, 80636 Munich, Germany \\ ${ }^{6}$ Institute of Physics, Federal University of Goiás, P. O. Box 131, 74001-970, Goiânia, Brazil
}

(Received 23 April 2020; accepted 28 July 2020; published 15 September 2020)

\begin{abstract}
The quench dynamics of many-body quantum systems may exhibit nonanalyticities in the Loschmidt echo, a phenomenon known as dynamical phase transition (DPT). Despite considerable research into the underlying mechanisms behind this phenomenon, several open questions still remain. Motivated by this, we put forth a detailed study of DPTs from the perspective of quantum phase space and entropy production, a key concept in thermodynamics. We focus on the Lipkin-Meshkov-Glick model and use spin-coherent states to construct the corresponding Husimi- $Q$ quasiprobability distribution. The entropy of the $Q$ function, known as Wehrl entropy, provides a measure of the coarse-grained dynamics of the system and, therefore, evolves nontrivially even for closed systems. We show that critical quenches lead to a quasimonotonic growth of the Wehrl entropy in time, combined with small oscillations. The former reflects the information scrambling characteristic of these transitions and serves as a measure of entropy production. On the other hand, the small oscillations imply negative entropy production rates and therefore signal the recurrences of the Loschmidt echo. Finally, we also study a Gaussification of the model based on a modified Holstein-Primakoff approximation. This allows us to identify the relative contribution of the low-energy sector to the emergence of DPTs. The results presented in this article are relevant not only from the dynamical quantum phase transition perspective but also for the field of quantum thermodynamics, since they point out that the Wehrl entropy can be used as a viable measure of entropy production.
\end{abstract}

DOI: 10.1103/PhysRevResearch.2.033419

\section{INTRODUCTION}

The dynamics of closed quantum many-body systems has been the subject of considerable interest since the early 2010s. After a sudden quench, for instance, the support of a local operator will in general spread through all of Hilbert space. The precise way through which this takes place reveals important information about the basic mechanisms underlying the dynamics. A particularly interesting example is the socalled dynamical phase transition (DPT), first discovered in Ref. [1], as far as we know, and subsequently explored in distinct situations. In Ref. [2] it was shown that DPTs have no connection with standard equilibrium phase transitions. The case of long-range interacting system was considered in Ref. [3], while Ref. [4] treated the case of nonintegrable systems. An attempt to classify DPTs, leading to the definition of first-order DPT, was introduced in Ref. [5], as far as we know.

Published by the American Physical Society under the terms of the Creative Commons Attribution 4.0 International license. Further distribution of this work must maintain attribution to the author(s) and the published article's title, journal citation, and DOI.
Finally, we can mention a very interesting connection between these DPTs and quantum speed limits, developed in Ref. [6]. On the experimental side, we can mention the observation of DPTs in the Ising model in an ion trap [7], in the topological phase in the Haldane model using optical lattices [8], and in a simulation of the Ising model in a quantum computer [9]. We refer the reader to the recent reviews [10-12] for further details about this field.

The central quantity in the theory of DPTs, which usually occur in a quenched quantum system, is the Loschmidt echo. The basic scenario consists of initially preparing a system in the ground state $\left|\psi_{0}\right\rangle$ of some Hamiltonian $H_{0}$. At $t=0$ the system is then quenched to evolve according to a different Hamiltonian $H$. The Loschmidt echo is defined as

$$
\mathcal{L}(t)=\left|\left\langle\psi_{0} \mid \psi_{t}\right\rangle\right|^{2}=\left|\left\langle\psi_{0}\left|e^{-i H t}\right| \psi_{0}\right\rangle\right|^{2},
$$

and therefore quantifies the overlap between the initial state and the evolved state at any given time. In other words, it measures how the support of the wave function spreads through the many-body Hilbert space.

In quantum critical systems, the Loschmidt echo in Eq. (1) is characterized by sharp recurrences [see Fig. 1(a) for an example]. The nature of these recurrences is more clearly 

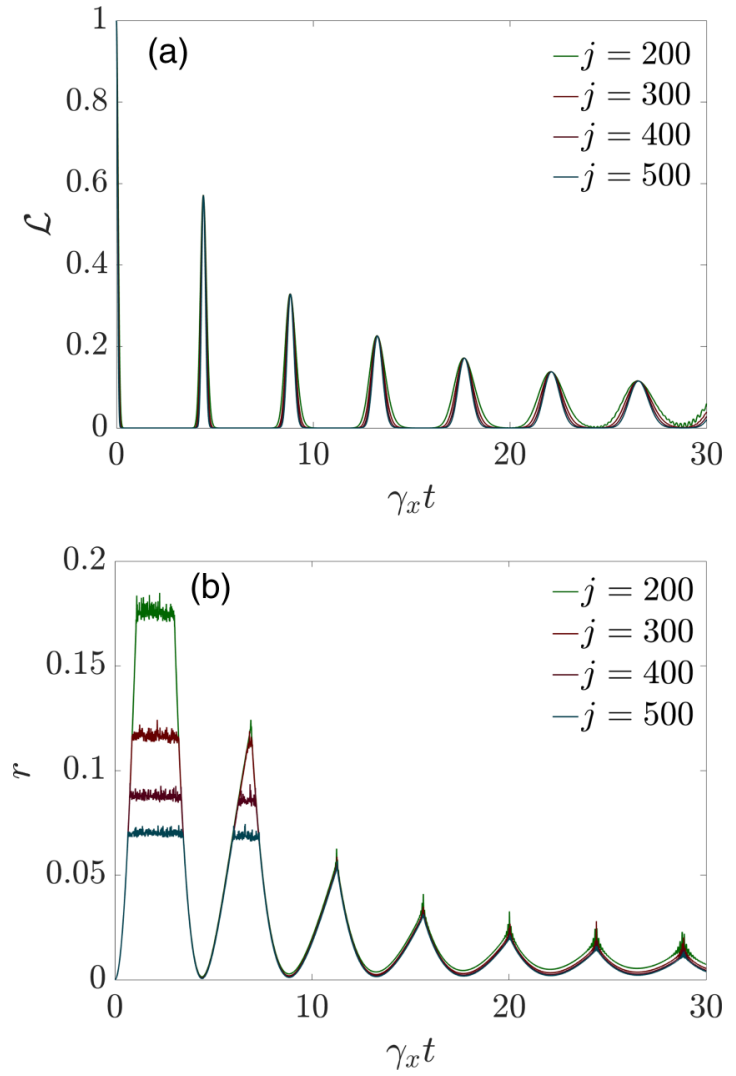

FIG. 1. Dynamical phase transition. The top panel shows the Loschmidt echo (1) while the bottom one displays the dynamical behavior of the rate function (2) for a quench in the LMG model, from $h_{0}=0$ to $h=0.8$. The curves correspond to different values of $j$. The plateaus that occur in $r(t)$ at short times is a numerical artifact. They happen because $\mathcal{L}(t)$ becomes exponentially small in these regions (even though this could be fixed with increasing the precision, this is not viable in practice because of the exponential dependence).

expressed in terms of the rate function,

$$
r(t)=-\frac{1}{N} \log \mathcal{L}(t),
$$

where $N$ is the system size. In the thermodynamic limit $(N \rightarrow$ $\infty)$, the rate $r(t)$ presents nonanalyticities (kinks) at certain instants of time [cf. Fig. 1(b)], which are the hallmark of DPTs. The Loschmidt echo in Eq. (1) shows a formal relation with a thermal partition function at imaginary time, which allows one to link these nonanalyticities to the Lee-YangFisher [13,14] zeros of $\mathcal{L}(t)$ (see Ref. [10] for more details).

Despite considerable progress in our understanding of DPTs, several open questions still remain, for instance, regarding their universality and if they can be captured from macroscopic properties [10-12]. In this article we focus on two deeply related questions. The first one concerns the transition from quantum to classical, i.e., which aspects of the problem are genuinely coherent and which could be understood from purely classical equations of motion. Defining this transition is not trivial for the great majority of models, such as spin chains. The second issue concerns which sectors of the Hamiltonian contribute to the transition. Indeed, quantum phase transitions depend only on the low-energy sector (ground state plus the first few excited states). DPTs, on the other hand, should in principle depend on the entire spectrum.

The interpretation of $\mathcal{L}(t)$ as a measure of how the support of the system spreads in time brings a clear thermodynamic flavour to DPTs. In the language of classical thermodynamics, an expanding gas fills all of available space, causing the entropy to grow monotonically in time. In closed quantum systems, however, the von Neumann entropy is a constant of motion, despite possible information scrambling. This idea has been explored with certain detail in the context of fluctuation theorems and nonequilibrium lag [15].

One way to reconcile these two views is to move to quantum phase space. In Ref. [16], the authors studied the dynamics of the Dicke model in terms of the Husimi- $Q$ quasiprobability function. The Husimi function contains the same amount of information as the system's density matrix but offers an alternative description that better captures the transition from quantum to classical. The authors of Ref. [16] showed that the closed (unitary) evolution yields, notwithstanding, a diffusive-type Fokker-Planck equation in phase space. It is, of course, a special type of diffusion in order to comply with the fact that the system is closed, and thus energy must be conserved. The Husimi function can be viewed as a convolution of the system's state with a heterodyne measurement. Consequently, it provides a coarse-grained description of the dynamics and thus naturally accounts for the scrambling of information.

The entropy associated with the $Q$ function is known as Wehrl's entropy $[17,18]$ and it can be attributed an operational meaning in terms of sampling through heterodyne measurements [19]. That is, the Wehrl entropy is the Shannon entropy associated with the probability distribution of heterodyne measurements. For this reason, it upper bounds the von Neumann entropy, since it encompasses also the extra Heisenberg uncertainty related to spin-coherent states. Moreover, for the same reason, the Wehrl entropy also evolves nontrivially even in closed system, unlike the von Neumann entropy, which is constant. It consequently captures the scrambling of information, very much like the Loschmidt echo in Eq. (1), but from the phase-space perspective. For these reasons, the Wehrl entropy serves as a useful quantifier of quantum dynamics and several nontrivial features associated with the transition from quantum to classical.

Motivated by this, we put forth in this article a detailed study of DPTs from the optics of quantum phase space. We focus on the Lipkin-Meshkov-Glick (LMG) model, describing the dynamics of a single macrospin [20-35] (Sec. II). This model allows for a well-defined classical limit, which takes place when the spin $j \rightarrow \infty$. In this case, the model reduces to the classical dynamics of a spinning top [29]. In addition, it allows for a neat construction in terms of quantum phase space by using the idea of spin-coherent states: The corresponding Husimi function describes a quasiprobability distribution in the unit sphere. Moreover, the LMG model has been employed in the study of DPT. Reference [36] builds a connection between two distinct definitions of dynamical critical behavior, a change in the order parameter and the singular behavior of the Loschmidt echo. Considering the finite-temperature case, a dynamical phase diagram was built in Ref. [37]. A 
semiclassical approach was employed in Ref. [38] in order to study the anomalous DPT. Finally, we mention the geometric perspective on DPT presented in Ref. [39].

As we show in Sec. III, the dynamics of the Wehrl entropy offers valuable insight about the nature of DPTs. After a critical quench, it grows quasimonotonically, combined with small oscillations. The growth reflects the information scrambling characteristic of DPTs. The oscillations, on the other hand, mimic the nonanalytic behavior of the Loschmidt echo and reflect information backflow to the initial Hilbert space sector. Finally, in Sec. IV, we also carry out an analysis using a generalized Holstein-Primakoff transformation that is known to faithfully capture the entire low-energy sector in the thermodynamic limit. This allows us to address which parts of the spectrum are essential for the description of DPTs. As we show, this procedure yields accurate predictions for small quenches but fails for the strong quenches required to observe DPTs.

\section{DYNAMICAL PHASE TRANSITION IN THE LIPKIN-MESHKOV-GLICK MODEL}

Let us consider the LMG model [20-22], described by the Hamiltonian

$$
H=-h J_{z}-\frac{1}{2 j} \gamma_{x} J_{x}^{2},
$$

where $j$ is the total angular momentum, $h \geqslant 0$ is the magnetic field, and $\gamma_{x}>0$ (critical field $h_{c}=\gamma_{x}$ ). This model can be viewed as the fully connected version of a system of $N=2 j$ spin- $1 / 2$ particles (it therefore presents mean-field exponents). Since it is analytically tractable, it has been the subject of several studies over the past decades [23-35].

\section{A. Brief review of the quantum phase transition}

Before describing the dynamical phase transition, let us firstly discuss the regular quantum phase transition for this model, which occurs in the thermodynamic limit, $j \rightarrow \infty$. In order to do this, it is convenient to define the so-called spin-coherent state,

$$
|\Omega\rangle=e^{-i \phi J_{z}} e^{-i \theta J_{y}}|j\rangle,
$$

where $|j\rangle$ is the eigenstate of $J_{z}$ with eigenvalue $j$ and $\theta \in$ $[0, \pi]$ and $\phi \in[0,2 \pi]$ are polar coordinates. These states represent the closest quantum analog of a classical angularmomentum vector of fixed length $j$, in the sense that the expectation values of the spin operators in the state of Eq. (4) take the form

$$
\left(\left\langle J_{x}\right\rangle,\left\langle J_{y}\right\rangle,\left\langle J_{z}\right\rangle\right)=j(\sin \theta \cos \phi, \sin \theta \sin \phi, \cos \theta) .
$$

Moreover, expectation values of higher powers, such as $\left\langle J_{x}^{2}\right\rangle$, differ from $\left\langle J_{x}\right\rangle^{2}$ only by terms which become negligible in the limit of large $j$. As a consequence, it can be shown that the leading order of the ground state of the LMG model in the thermodynamic limit is a spin-coherent state for certain values of $\theta$ and $\phi$ [29]. The energy in this limit can be computed as $E=\langle\Omega|H| \Omega\rangle$, resulting in [40]

$$
\frac{E}{j}=-h \cos \theta-\frac{\gamma_{x}}{2} \sin ^{2} \theta \cos ^{2} \phi .
$$

The corrections to this behavior will be computed explicitly in Sec. IV.

The ground-state energy is then found by minimizing Eq. (5) over $\theta$ and $\phi$, leading to the set of equations

$$
\begin{aligned}
\sin \theta\left(h-\gamma_{x} \cos \theta \cos ^{2} \phi\right) & =0, \\
\gamma_{x} \sin ^{2} \theta \cos \phi \sin \phi & =0 .
\end{aligned}
$$

For $h>\gamma_{x}$ the only solution is $\theta=0$, in which case $\phi$ is arbitrary. For $h<\gamma_{x}$, however, two new solutions appear, corresponding to

$$
\cos \theta=\frac{h}{\gamma_{x}},
$$

and $\phi=0$ or $\phi=\pi$. The magnetization $m=\sin \theta \cos \phi$ therefore serves as the order parameter of the model. This is identically zero for $h>\gamma_{x}$ and $m=\sqrt{h_{c}^{2}-h^{2}} / \gamma_{x}$ otherwise. The emergence of these new solutions identifies the critical field $h_{c}=\gamma_{x}$.

\section{B. Dynamical phase transition}

Let us now consider the introduction of quenches in the field $h$. The system is prepared in the ground state $\left|\psi_{0}\right\rangle$ of $H_{0}=H\left(h_{0}\right)$, and at $t=0$, it evolved under the final Hamiltonian $H=H(h)$. To quantify the DPT, we use the Loschmidt echo defined in Eq. (1) and the corresponding rate in Eq. (2), with $N=2 j$. In this model, a subtlety arises because the ground state is twofold degenerate. As discussed in Appendix $\mathrm{B}$, however, this introduces effects which become negligible in the thermodynamic limit. For this reason, we henceforth focus only on the analysis starting from one of the ground states.

For the sake of concreteness, we focus on quenches from $h_{0}=0$ to $h<\gamma_{x}$. Results for the Loschmidt echo and the rate function are shown in Fig. 1 for several values of $j$. The echo (top panel in Fig. 1) vanishes for certain periods of time but presents sharp periodic revivals at certain instants. This is convoluted with a damping, causing the time decay of the magnitude of $\mathcal{L}(t)$. The presence of a DPT becomes visible in the rate function (bottom panel in Fig. 1), which presents kinks at certain instants of time, called critical times $t_{c}$.

\section{ENTROPIC DYNAMICS IN QUANTUM PHASE SPACE}

Let us now address the core part of our article, in which we put forth an analysis of the DPT in Fig. 1 from the perspective of quantum phase space. The spin Husimi- $Q$ function associated with an arbitrary density matrix $\rho$ is defined as

$$
Q(\Omega)=\langle\Omega|\rho| \Omega\rangle,
$$

where $|\Omega\rangle$ are the spin-coherent states given in Eq. (4). This quantity is always nonnegative and normalized to unity according to $(2 j+1) / 4 \pi \int d \Omega Q(\Omega)=1$, where $d \Omega=$ $\sin \theta d \theta d \phi$. It therefore represents a quasiprobability distribution in the unit sphere, offering the perfect platform to understand the quantum to classical transition [41]. In our case, $\rho=\left|\psi_{t}\right\rangle\left\langle\psi_{t}\right|$ so the Husimi function simplifies to $Q=$ $\left|\left\langle\Omega \mid \psi_{t}\right\rangle\right|^{2}$. 
The entropy associated with $Q(\Omega)$ is known as Wehrl's entropy $[17,18]$, defined as

$$
S_{Q}=-\frac{2 j+1}{4 \pi} \int d \Omega Q(\Omega) \ln Q(\Omega) .
$$

An operational interpretation of this quantity in terms of sampling through heterodyne measurements was given in Ref. [19]. It has also been applied in different contexts, like entanglement theory [42], uncertainty relations [43], and quantum phase transitions [44], just to name a few. In the context of thermodynamics, our interest here, a theory of entropy production for spin systems was put forth in Ref. [45] and the corresponding bosonic analog in Ref. [46].

In classical thermodynamics, the entropy of a closed system should be monotonically increasing with time. The quantity

$$
\frac{d S_{Q}}{d t}:=\Pi_{Q}
$$

is interpreted as the entropy production rate, since it reflects the entropy that is irreversibly produced in the system. The second law then states that $\Pi_{Q} \geqslant 0$. In open systems, on the other hand, one has instead

$$
\frac{d S_{Q}}{d t}=\Pi_{Q}-\Phi_{Q},
$$

where $\Phi_{Q}$ is the entropy flux rate from the system to the environment. In open systems, $d S_{Q} / d t$ does not have a welldefined sign, since the flux $\Phi_{Q}$ can be arbitrary. However, one still has $\Pi_{Q} \geqslant 0$. In the present case there is no associated flux $\left(\Phi_{Q}=0\right)$, since the dynamics of the system is closed. Moreover, it is not guaranteed that $d S_{Q} / d t \geqslant 0$ for all times, since our system is not in the thermodynamic limit. Notwithstanding, one still expects that strong information scrambling, as happens in critical systems, should lead to a $\Pi_{Q}$ which is most of the time nonnegative.

Numerical results for the Wehrl entropy in Eq. (8) and the entropy production rate in Eq. (9) are shown in Fig. 2 for the same quench protocol used in Fig. 1, which are remarkable. As $j$ increases, the Wehrl entropy presents small oscillations enveloping a monotonically increasing behavior. This increase clearly reflects the information scrambling characteristic of the DPT. It shows that, as time passes, the coarse-grained nature of $Q(\Omega)$ causes the available information about the system's state to be degraded as a function of time. For long times and $j$ sufficiently large, $S_{Q}$ has a tendency to saturate at a constant value. As anticipated, $\Pi_{Q}$ oscillates in time, being predominantly positive, but also becoming negative at certain times. These negativities represent the backflow of information, which is characteristic of the recurrences in $\mathcal{L}(t)$ (see Fig. 1).

Figure 3 shows the entropy production rate $\Pi_{Q}$ along with the rate function $r(t)$ for $j=300$. As we can see, their behaviors are clearly linked. In order to make such relation clearer, we present in Fig. 4 the relation between the critical times $t_{c}$ where the dynamical quantum phase transitions occur, i.e., the nonanalytical points of $r(t)$, and the times $t_{m}$ at which $\Pi_{Q}$ present local maxima. From this result it becomes clear that, for large $j$, one approaches $t_{c} \approx t_{m}$, showing how the maxima of $\Pi_{Q}$ perfectly correlate with the critical times. This
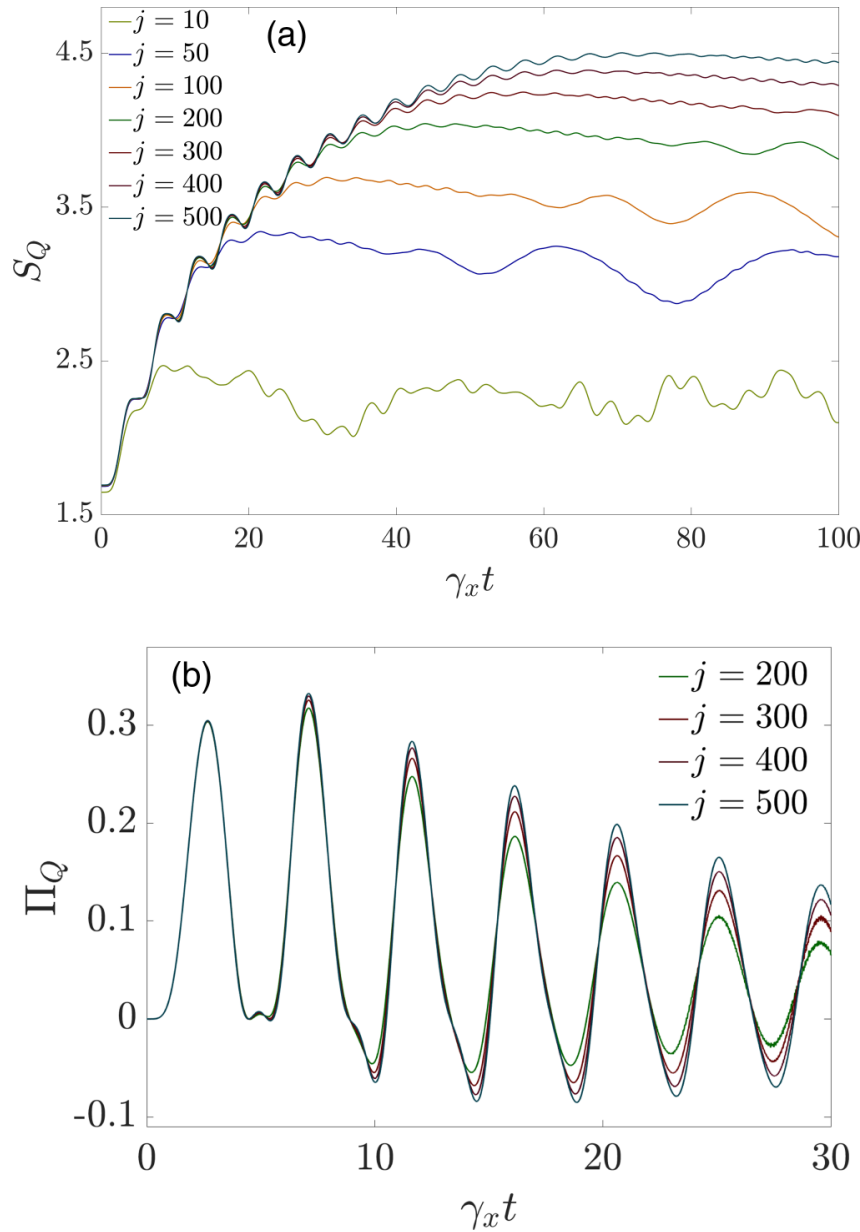

FIG. 2. Entropy dynamics. The top panel shows the dynamics of the Wehrl entropy (8) while the entropy production rate, Eq. (9), is displayed in the bottom panel, for the same quench protocol used in Fig. 1.

corroborates the idea that the oscillations in $\Pi_{Q}$ indeed reflect the DPT. A more detailed analysis is presented in Appendix B.

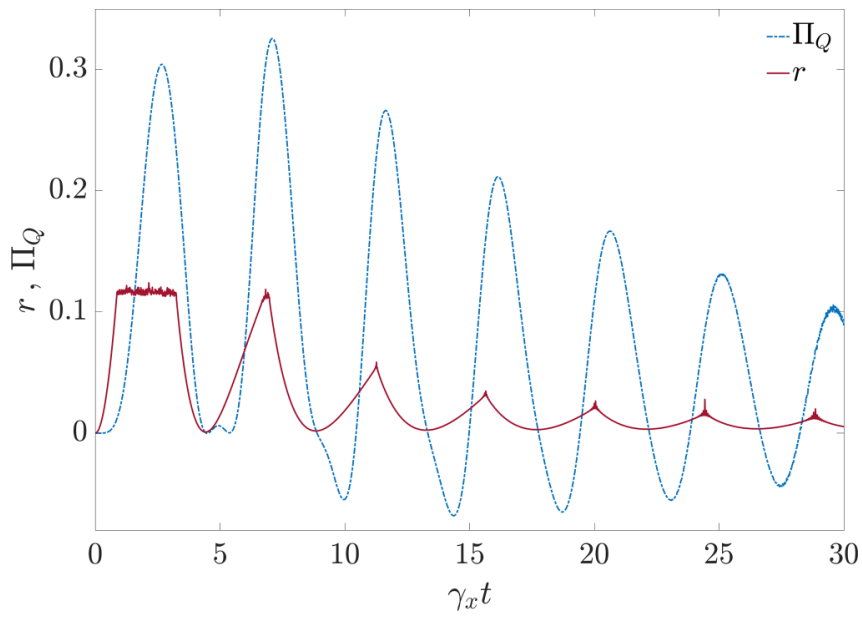

FIG. 3. Rate function $r(t)$, along with the entropy production rate $\Pi_{Q}$, for $j=300$. Other parameters are the same as in Fig. 1 . 


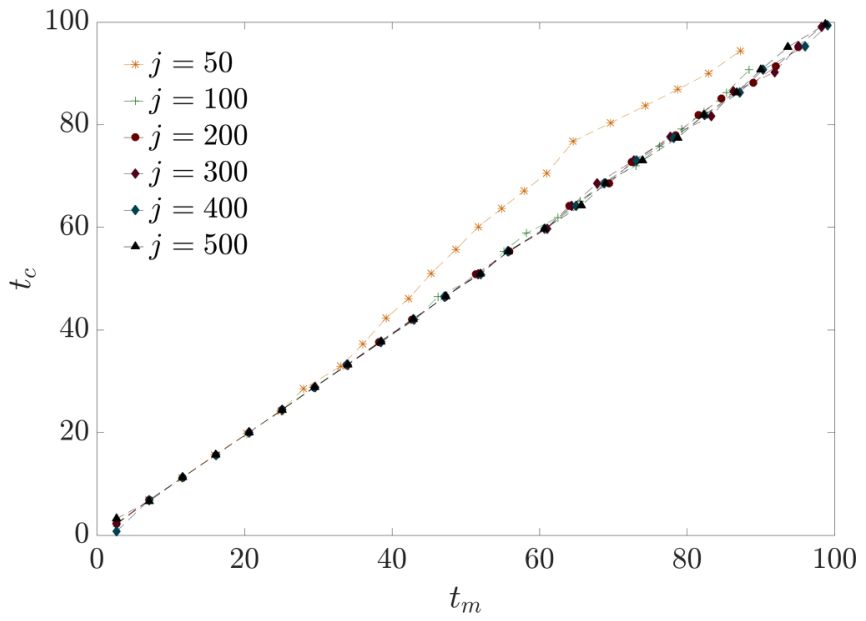

FIG. 4. Critical times $t_{c}$, associated with the nonanalytical behavior of $r(t)$ and the times $t_{m}$ corresponding to local maxima of $\Pi_{Q}$. Other parameters are the same as in Fig. 1.

Finding a closed-form expression for the entropy production rate (9) is in general not possible. Notwithstanding, we were able to identify which parts of the dynamics, in terms of the associated Fokker-Planck equation, contribute to $\Pi_{Q}$. This analysis, however, is cumbersome and it is thus postponed to Appendix A.

We have performed several numerical studies regarding the role of different quenches and we were unable to observe any effect on the connection between the Wehrl entropy and the DPT. By changing the value of $h$ the quantitative values of the considered physical quantities change, as shown in the Appendix, but the qualitative behavior is the same. Therefore, the linear behavior observed in Fig. 4 still holds. We start with $h_{0}=0$ due to the fact that we need a degenerate ground state in order to observe the DPT, which is clearly achieved for this value of the field.

\section{CONTRIBUTION FROM THE LOW-ENERGY SECTOR}

\section{A. Holstein-Primakoff approximation for the LMG model}

Equilibrium quantum phase transitions are almost entirely described by the low-energy sector, i.e., ground state plus the first few excited states. This is not true for DPTs which, in principle, depend on the entire spectrum. In this section we ask which aspects of DPTs can nonetheless be captured by the low-energy sector. We do this by introducing a Gaussification procedure based on a generalized Holstein-Primakoff (HP) representation [47]. Finally, we then compare the predictions of this HP model with the full numerics studied in the previous section.

The HP method represents spin operators in terms of a single bosonic mode by means of the nonlinear transformation [47],

$$
\begin{aligned}
J_{z} & =j-a^{\dagger} a, \\
J_{+} & =\sqrt{2 j-a^{\dagger} a} a,
\end{aligned}
$$

where $a$ is a bosonic operator satisfying $\left[a, a^{\dagger}\right]=1$. Equation (11a) shows that the excitations of $a^{\dagger} a$ are mapped onto excitations of $J_{z}$, starting from the state $|j\rangle$, downwards. This is reasonable when $h>h_{c}$. But when $h<h_{c}$, the ground state will not be close to $|j\rangle$ at all. To take this into account, we first rotate the Hamiltonian (3) by an angle $\theta$ around the $y$ axis, before applying the HP transformation. That is, we first consider the rotated Hamiltonian

$$
\begin{aligned}
H_{R}= & e^{i \theta S_{y}} H e^{-i \theta S_{y}} \\
= & -h\left(J_{z} \cos \theta-J_{x} \sin \theta\right) \\
& -\frac{\gamma_{x}}{2 j}\left(J_{z} \sin \theta+J_{x} \cos \theta\right)^{2},
\end{aligned}
$$

where the value of $\theta$ will be fixed below. We now introduce the HP transformation on $H_{R}$ instead of $H$. Expanding for large $j$ and keeping only terms which are at most quadratic in $a$ and $a^{\dagger}$, one finds

$$
\begin{aligned}
H_{R}= & E-\frac{\sqrt{2 j}}{2} \sin \theta\left(\gamma_{x} \cos \theta-h\right)\left(a+a^{\dagger}\right) \\
& +a^{\dagger} a\left(\gamma_{x} \sin ^{2} \theta+h \cos \theta\right) \\
& -\frac{\gamma_{x}}{4} \cos ^{2} \theta\left(a+a^{\dagger}\right)^{2},
\end{aligned}
$$

where $E$ is the classical energy given in Eq. (5) with $\phi=0$. The role of $\phi$ is trivial, it is not necessary to rotate around the $z$ axis as well.

We can now choose $\theta$ to eliminate the linear term proportional to $a+a^{\dagger}$. This leads to the same choice that minimized the classical energy in Eq. (6), i.e.,

$$
\theta_{h}=\left\{\begin{array}{ll}
\arccos \left(h / \gamma_{x}\right), & \text { if } h<h_{c}=\gamma_{x} . \\
0 & \text { otherwise }
\end{array} .\right.
$$

With this choice, Eq. (13) reduces to

$$
H_{R}(h)=E+\gamma_{x} a^{\dagger} a-\frac{h^{2}}{4 \gamma_{x}}\left(a+a^{\dagger}\right)^{2}, \quad h<h_{c},
$$

and

$$
H_{R}(h)=E+h a^{\dagger} a-\frac{\gamma_{x}}{4}\left(a+a^{\dagger}\right)^{2}, \quad h>h_{c} .
$$

Therefore, the HP method leads to the same classical energy landscape as using spin-coherent states [Eq. (5)]. However, it is important to remark that it yields an operator-based representation of the fluctuations around the ground state. Note how $E \sim j$ is extensive, whereas the fluctuations in Eqs. (15) and (16) are independent of $j$. Notwithstanding, as we will find below, this does not mean the fluctuations are negligible when they become significantly close to criticality.

We now use the above results to determine the ground state and the energy gap between the ground state and the first excited state. To do this, we introduce the squeeze operator $S_{\xi}=e^{\frac{\xi}{2}\left(a^{2}-\left(a^{\dagger}\right)^{2}\right)}$, where for our purposes it suffices to take $\xi>0$. This allows us to write Eqs. (15) and (16), to constants, as

$$
H_{R}(h)=E+\omega_{h} S_{h}^{\dagger} a^{\dagger} a S_{h},
$$

where

$$
\omega_{h}= \begin{cases}\sqrt{\gamma_{x}^{2}-h^{2}}, & \text { if } h<h_{c} \\ \sqrt{h\left(h-\gamma_{x}\right)}, & \text { otherwise }\end{cases}
$$


and $S_{h}=S\left(\xi_{h}\right)$ with

$$
\xi_{h}= \begin{cases}-\frac{1}{4} \ln \left(1-h^{2} / \gamma_{x}^{2}\right), & \text { if } h<h_{c}, \\ -\frac{1}{4} \ln \left(1-\gamma_{x} / h\right), & \text { otherwise. }\end{cases}
$$

The Hamiltonian (17) is now diagonal, so that $\omega_{h}$ describes precisely the energy level spacing of the first few excited levels. Whence, we conclude that, at low energies, the excitations are equally spaced with energy gap $\omega_{h}$. As a feature of quantum phase transitions, the gap closes at $h=h_{c}$. Moreover, it does so from both directions and in a manner which is not symmetric in $h$.

Finally, to compute the ground state, we must first go back to the original Hamiltonian by undoing the rotation in Eq. (12). In the language of the HP transformation (11b), a rotation around $y$ becomes a displacement of the bosonic mode $a$,

$$
e^{i \theta J_{y}}=D\left(\alpha_{h}\right)=e^{\alpha_{h} a^{\dagger}-\alpha_{h}^{*} a},
$$

where $\alpha_{h}=-\sqrt{2 j} \theta_{h} / 2$ and $\theta_{h}$ is given in Eq. (14). By combining Eqs. (12) and (17), then it yields the original Hamiltonian in the form

$$
H=D_{h}^{\dagger} S_{h}^{\dagger}\left[\omega_{h} a^{\dagger} a\right] S_{h} D_{h},
$$

where $D_{h}=D\left(\alpha_{h}\right)$. The ground state is now readily found to be

$$
\left|\psi_{\mathrm{gs}}(h)\right\rangle=D_{h}^{\dagger} S_{h}^{\dagger}|0\rangle
$$

where $|0\rangle$ is the vacuum defined by $a$. The ground state is consequently a displaced squeezed state. The amount of displacement, $\alpha_{h}=-\sqrt{2 j} \theta_{h} / 2$ is zero when $h>h_{c}$ and nonzero otherwise. Moreover, the displacement scales as $\sqrt{j}$ and thus become extremely large in the thermodynamic limit. This displacement is precisely the prediction using spin-coherent states but written in a bosonic language. In addition, the state is also squeezed by the amount $\xi_{h}$ given in Eq. (19). This therefore represents a correction on top of the spin-coherent state predictions. The squeezing is $j$ independent but diverges at criticality. Thus, very close to the critical point, the state should differ significantly from a spin-coherent state.

\section{B. Loschmidt echo}

Armed with the aforementioned results, we can now readily compute both the Loschmidt echo and the rate function within the HP approximation. The system is initially prepared in the ground state $\left|\psi_{\mathrm{gs}}\left(h_{0}\right)\right\rangle$ corresponding to a field $h_{0}$. After the quench, the field changes to $h$. Since $S_{h}$ and $D_{h}$ are unitary, the time-evolution operator can be written as

$$
e^{-i H t}=D_{h}^{\dagger} S_{h}^{\dagger} e^{-i \omega_{h} t a^{\dagger} a} S_{h} D_{h} .
$$

The evolved state at time $t$ will then be

$$
\left|\psi_{t}\right\rangle=e^{-i H t}\left|\psi_{0}\right\rangle=D_{h}^{\dagger} S_{h}^{\dagger} e^{-i \omega_{h} t a^{\dagger} a} S_{h} D_{h} D_{h_{0}}^{\dagger} S_{h_{0}}^{\dagger}|0\rangle .
$$

The Loschmidt echo $\mathcal{L}(t)=\left|\left\langle\psi_{0} \mid \psi_{t}\right\rangle\right|^{2}$ becomes

$$
\mathcal{L}(t)=\left|\left\langle 0\left|S_{h_{0}} D_{h_{0}} D_{h}^{\dagger} S_{h}^{\dagger} e^{-i \omega_{h} t a^{\dagger} a} S_{h} D_{h} D_{h_{0}}^{\dagger} S_{h_{0}}^{\dagger}\right| 0\right\rangle\right|^{2} .
$$

This can be simplified by exploiting the algebra of displacement and squeeze operators, which in this case is facilitated by the fact that the arguments $\alpha_{h}$ and $\xi_{h}$ are all
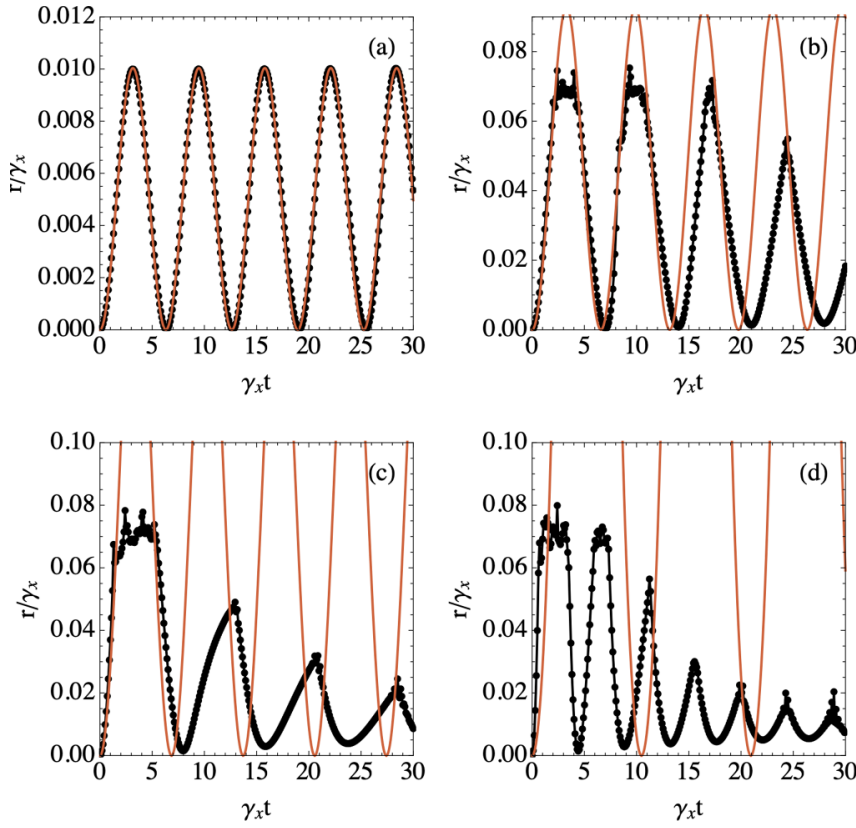

FIG. 5. Comparison of the rate function $r(t)$ between the full numerics (black points) and the Holstein-Primakoff approximation [Eq. (27)] for $j=200$. Each curve describes a quench from $h_{0}=0$ to (a) $h / \gamma_{x}=0.1$, (b) $h / \gamma_{x}=0.3$, (c) $h / \gamma_{x}=0.4$, and (d) $h / \gamma_{x}=0.8$ [cf. Fig. 1(b)].

real. First, one has $D_{h} D_{h_{0}}^{\dagger}=D(\delta \alpha)$, where $\delta \alpha=-\sqrt{2 j}\left(\theta_{h}-\right.$ $\left.\theta_{h_{0}}\right) / 2$. Second, $D(\delta \alpha) S_{h_{0}}^{\dagger}=S_{h_{0}}^{\dagger} D(\delta \tilde{\alpha})$, where $\delta \tilde{\alpha}=\delta \alpha e^{-\xi_{h_{0}}}$. Finally, we combine the two squeezing operations as $S_{h} S_{h_{0}}^{\dagger}=$ $S(\delta \xi)$, where $\delta \xi=\xi_{h}-\xi_{h_{0}}$. This leads to

$$
\mathcal{L}(t)=\left|\left\langle 0\left|D^{\dagger}(\delta \tilde{\alpha}) S^{\dagger}(\delta \xi) e^{-i \omega_{h} t a^{\dagger} a} S(\delta \xi) D(\delta \tilde{\alpha})\right| 0\right\rangle\right|^{2} .
$$

This expression can now be evaluated by noting that it represents the vacuum expectation of a thermal squeezed displaced Gaussian state at imaginary temperature $\beta=-i \omega_{h}$. The result is therefore

$$
\mathcal{L}(t)=\frac{\exp \left\{-\frac{2 j\left(\theta_{h}-\theta_{h_{0}}\right) e^{-2 \xi_{h_{0}}}}{1+4 e^{\xi \xi} \cot ^{2}\left(\omega_{h} t / 2\right)}\right\}}{\sqrt{\cos ^{2}\left(\omega_{h} t\right)+\cosh ^{2}(2 \delta \xi) \sin ^{2}\left(\omega_{h} t\right)}},
$$

an expression which depends only on the HP parameters $\theta_{h}$, $\xi_{h}$, and $\omega_{h}$, given by Eqs. (14), (18), and (19), respectively.

Note also how the exponent in (26) is extensive in $j$. As a consequence, the rate function (2) with $N=2 j$ becomes, in the thermodynamic limit,

$$
r(t)=\frac{\left(\theta_{h}-\theta_{h_{0}}\right) e^{-2 \xi_{h_{0}}}}{1+4 e^{\delta \xi} \cot ^{2}\left(\omega_{h} t / 2\right)} .
$$

We can now finally address the main question posed in the beginning of this section. Namely, what is the contribution of the low-energy sector to DPTs. To do this, we simply compare Eq. (27) with the full numerics. The results are presented in Fig. 5. They clearly that DPTs cannot be explained making use only of the low-energy sector. In Fig. 5(a), for instance, we compare quenches from $h_{0}=0$ to $h / \gamma_{x}=0.1$. In this case, Eq. (27) (in red) faithfully captures the physics of the full numerical solution (black points). However, for these small 
quenches, the nonanalyticities of $r(t)$ are not yet present. Conversely, as the value of $h$ increases, the signature kinks of the DPTs start to appear, whereas Eq. (27) remains perfectly analytical. In fact, Eq. (27) can only become nonanalytic when $h=\gamma_{x}=1$, in which case $\omega_{h} \rightarrow 0$ and the cotangent diverges. At this point, the high-energy sector becomes so important that, albeit nonanalytic, Eq. (27) cannot capture at all the physics of the problem.

\section{CONCLUSION}

In summary, we studied the dynamical behavior of the entropy production in a closed system that undergoes a dynamical quantum phase transition. Specifically, we considered sudden quenches in the Lipkin-Meshkov-Glick model under the perspective of the Husimi- $Q$ quasiprobability distribution. Such an approach allowed us to define the entropy associated with the $Q$ function, known as Wehrl entropy, which is a measure of the coarse-grained dynamics of the system.

From such phase-space approach, we were able to show that critical quenches lead to a quasimonotonic growth of the Wehrl entropy in time, thus demonstrating the scrambling of quantum information, a characteristic feature of these transitions. Moreover, the entropy rate also presents small oscillations, implying negative entropy production rates at certain instants, which signals the recurrences of the Loschmidt echo. These results are relevant not only from the dynamical quantum phase transition perspective but also for the field of quantum thermodynamics, since they point out that the Wehrl entropy can be used as a viable measure of entropy production.

Note that since both features observed here-the tendency for the entropy to monotonically increase and the small oscillations in its time evolution-are consequences of the information degradation due to scrambling and the recurrences presented in the Loschmidt echo, respectively. Since these features directly reflects the dynamics of the Loschmidt echo in any system presenting DPT, we expect that our conclusions
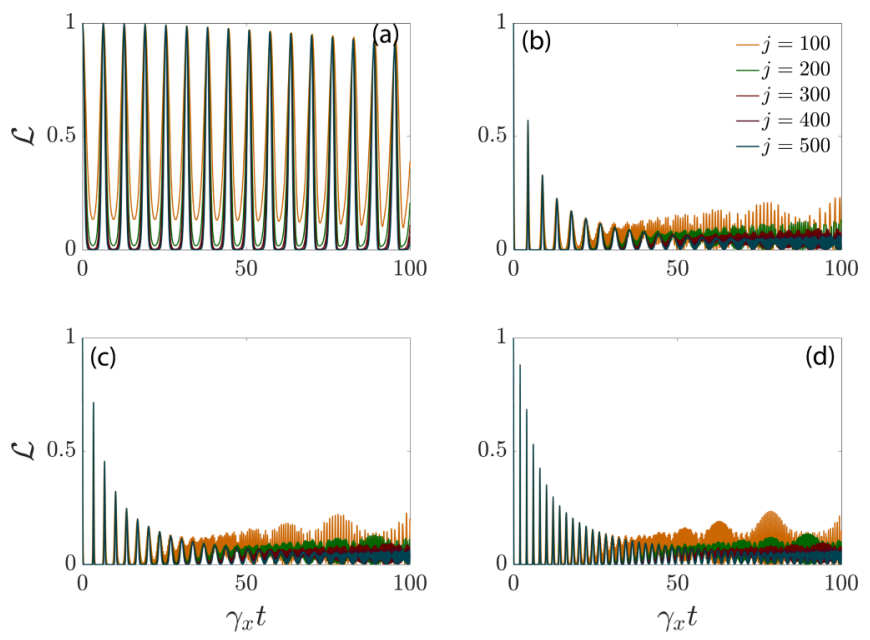

FIG. 6. Loschmidt echo for the first ground state. From top to bottom and from left to right we consider quenches from $h_{0}=0$ to $h=0.1,0.8,1.0$, and 1.6.
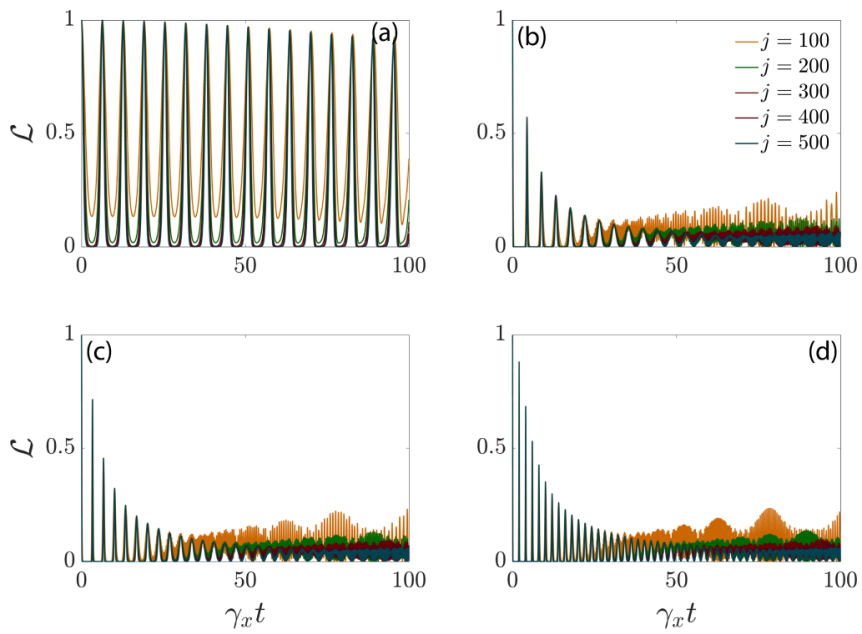

FIG. 7. Loschmidt echo for the second ground state. From top to bottom and from left to right we consider quenches from $h_{0}=0$ to $h=0.1,0.8,1.0$, and 1.6.

apply to the DPT itself, not reflecting any particular characteristic of the considered model.

The dynamical behavior of the entanglement entropy was experimentally addressed in Ref. [7] for the LMG model using an ion trap setup. It is interesting to observe that production of entanglement, as measured by the von Neuman entropy of the reduced state (half of the chain in the present case) resembles the one observed here for the Wehrl entropy. The von Neumann entropy cannot be identified with the thermodynamic entropy for systems out of equilibrium, as in the present case and, due to its coarse-grained nature, we claim that the Wehrl entropy is the natural candidate for developing a thermodynamic theory for DPT. However, an interesting connection between entanglement monotones and Wehrl entropy was discovered in Ref. [42] and further studies on these lines could lead to a deeper connection between thermodynamics and entanglement.
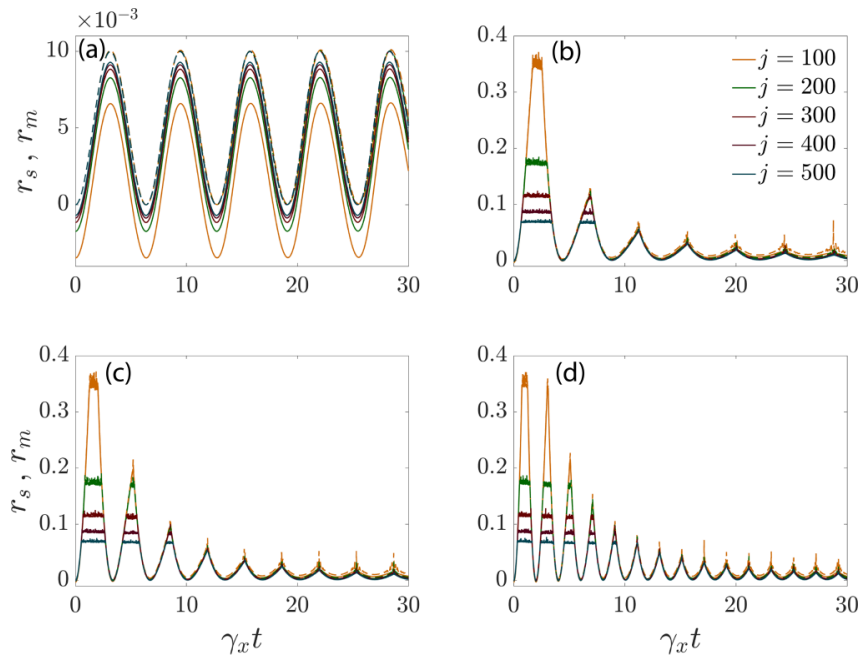

FIG. 8. Comparison between $r$ (solid lines) and $r_{m}$ (dashed lines). From top to bottom and from left to right we consider quenches from $h_{0}=0$ to $h=0.1,0.8,1.0$, and 1.6. 

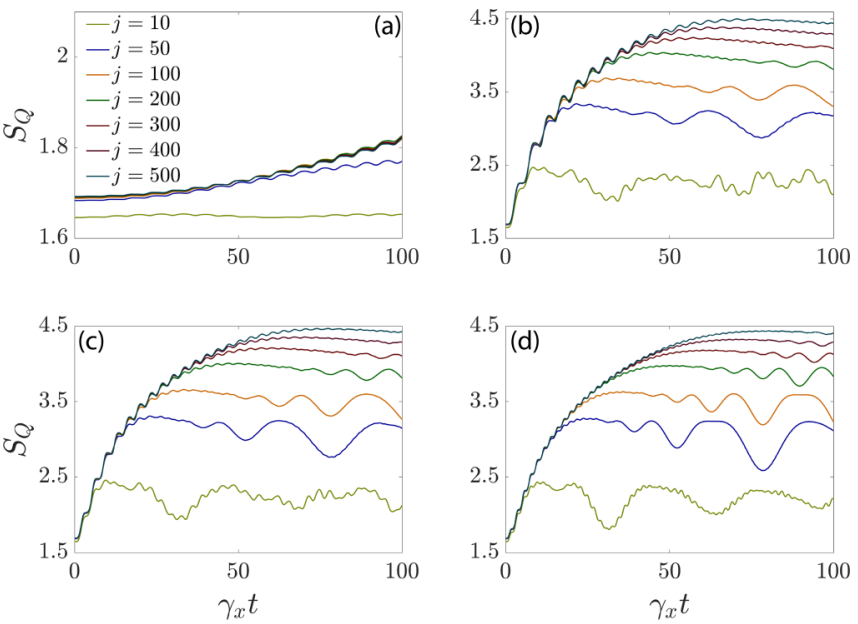

FIG. 9. Entropy for the first ground state. From top to bottom and from left to right we consider quenches from $h_{0}=0$ to $h=0.1,0.8$, 1.0, and 1.6.

Finally, based on a modified Holstein-Primakoff approximation, a type of Gaussification method, we addressed the contribution of the low-energy sector to the dynamical quantum phase transition. This procedure, which is known to faithfully capture the entire low-energy sector in the thermodynamic limit, fails to accurately predict the behavior of the system under dynamical quantum phase transitions.

Despite considerable research into the underlying mechanisms behind this phenomenon, several open questions still remain and we believe that the phase-space perspective that we put forth here may contribute to deepen our understanding, specially regarding the thermodynamics of such phase transitions, as indicated by the strong connection between critical times, characterizing the critical transition, and the entropy production rate, a key concept in thermodynamics.
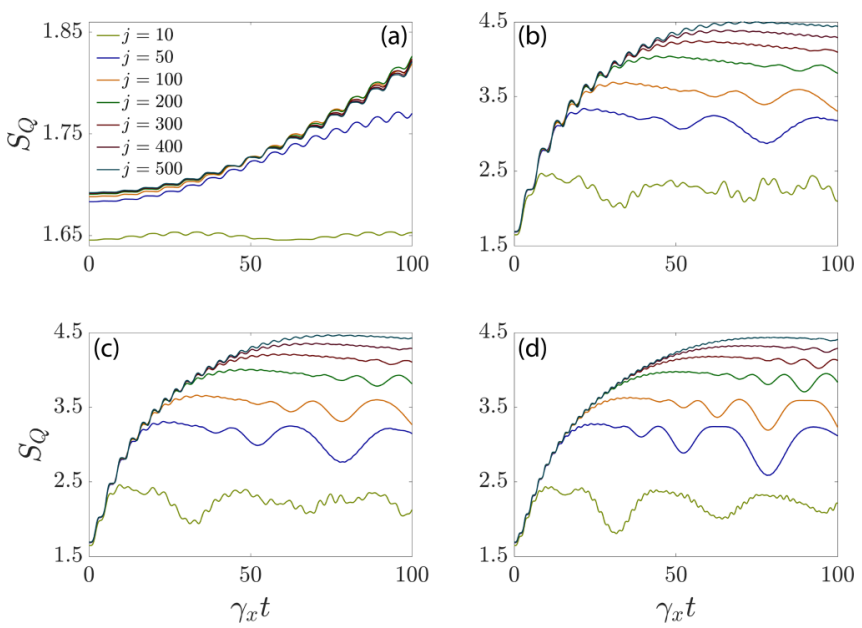

FIG. 10. Entropy for the second ground state. From top to bottom and from left to right we consider quenches from $h_{0}=0$ to $h=0.1$, $0.8,1.0$, and 1.6.
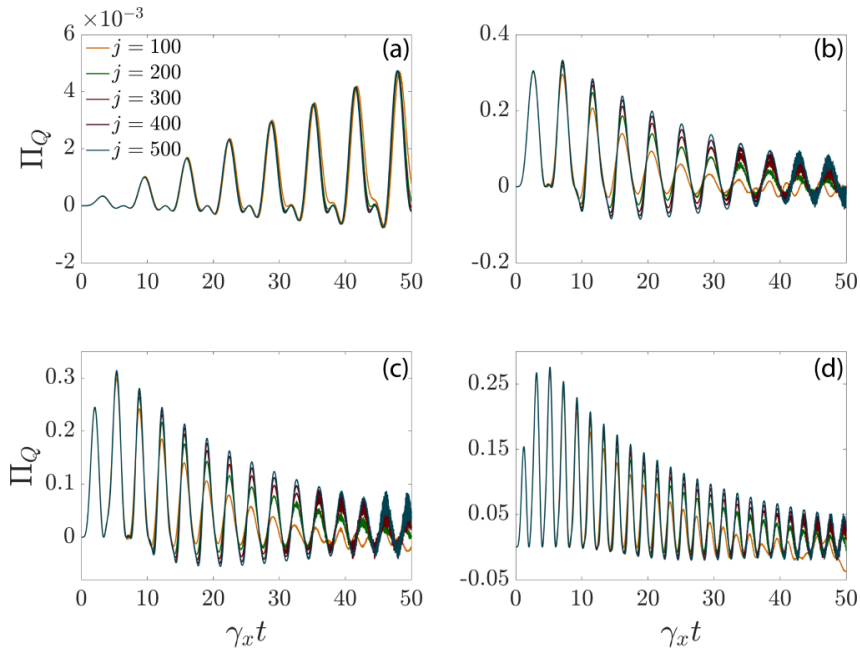

FIG. 11. Entropy production rate for the first ground state. From top to bottom and from left to right we consider quenches from $h_{0}=$ 0 to $h=0.1,0.8,1.0$, and 1.6.

\section{ACKNOWLEDGMENTS}

E.S., M.S., and L.C.C. acknowledge support from Spanish MCIU/AEI/FEDER (Grant No. PGC2018-095113-BI00), Basque Government IT986-16, Projects No. QMiCS (820505) and No. OpenSuperQ (820363) of the EU Flagship on Quantum Technologies, EU FET Open Grant Quromorphic (828826), the U.S. Department of Energy, Office of Science, Office of Advanced Scientific Computing Research (ASCR) quantum algorithm teams program, under field work proposal number ERKJ333, and the Shanghai STCSM (Grant No. 2019SHZDZX01-ZX04). L.C.C. also thanks the Brazilian Agencies CNPq, FAPEG. L.C.C. and G.T.L. acknowledge the Brazilian National Institute of Science and Technology of Quantum Information (INCT/IQ) for the financial support. This study was financed in part by the Coordenação de Aperfeiçoamento de Pessoal de Nível Superior, Brasil (CAPES),
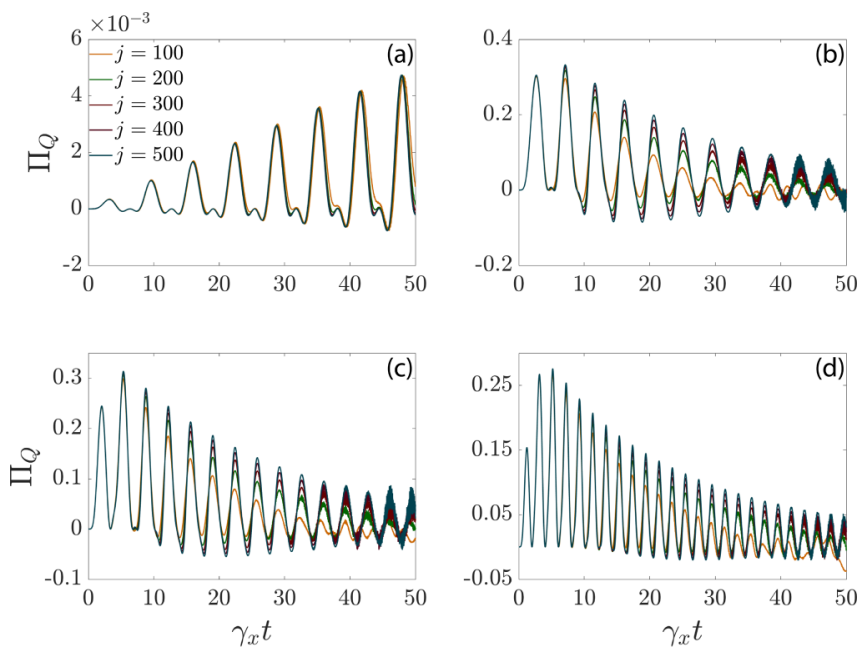

FIG. 12. Entropy production rate for the second ground state. From top to bottom and from left to right we consider quenches from $h_{0}=0$ to $h=0.1,0.8,1.0$, and 1.6. 
Finance Code 001. G.T.L. acknowledges the support from the São Paulo Research Foundation FAPESP (Grants No. 2017/07973-5, No. 2017/50304-7, and No. 2018/12813-0). B.O.G. acknowledges the warm hospitality of QUTIS Center where this work was developed.

\section{APPENDIX A: ENTROPY PRODUCTION FOR LMG MODEL}

In this Appendix we analytically compute the entropy production rate for the LMG model, Eq. (3). To accomplish this task, we use the Schwinger map to transform spin operators into bosonic operators $[45,48]$. This map employs two sets of bosonic operators, $a$ and $b$, such that

$$
\begin{aligned}
& J_{z}=\frac{1}{2}\left(a^{\dagger} a-b^{\dagger} b\right) \\
& J_{+}=J_{-}^{\dagger}=a^{\dagger} b .
\end{aligned}
$$

To simplify notation we will employ the bosonic coherent state representation for the Husimi function. In this way, we define $\alpha$ and $\beta$ as the complex amplitudes associated with operators $a$ and $b$, respectively. The correspondence table between bosonic operator acting on a state $\rho$ and a differential operator acting on the Husimi $Q$ function $Q(\alpha, \beta)$ is

$$
\begin{aligned}
o_{i} \rho & \rightarrow\left(\mu_{i}+\partial_{\bar{\mu}_{i}}\right) Q\left(\mu_{i}, \bar{\mu}_{i}\right) \\
o_{i}^{\dagger} \rho & \rightarrow \bar{\mu}_{i} Q\left(\mu_{i}, \bar{\mu}_{i}\right) \\
\rho o_{i} & \rightarrow \mu_{i} Q\left(\mu_{i}, \bar{\mu}_{i}\right) \\
\rho o_{i}^{\dagger} & \rightarrow\left(\bar{\mu}_{i}+\partial_{\mu_{i}}\right) Q\left(\mu_{i}, \bar{\mu}_{i}\right),
\end{aligned}
$$

where $o_{i}$ stands for $a$ or $b$ and $\mu_{i}$ represents either $\alpha$ or $\beta, \bar{\mu}_{i}$ denotes complex conjugation. Using Eqs. (A1) and (A2) one finds

$$
\left[J_{z}, \rho\right] \rightarrow \mathcal{J}_{z}=\frac{1}{2}\left[\left(\alpha \partial_{\alpha} Q-\bar{\alpha} \partial_{\bar{\alpha}} Q\right)-\left(\beta \partial_{\beta} Q-\bar{\beta} \partial_{\bar{\beta}} Q\right)\right],
$$

and, since $2 J_{x}=J_{+}+J_{-}$, it follows that

$$
\begin{aligned}
& 4\left[J_{x}^{2}, \rho\right] \rightarrow 4 \mathcal{J}_{x^{2}} \\
& \quad=\left(2|\alpha|^{2}+1\right)\left(\bar{\beta} \partial_{\bar{\beta}} Q-\beta \partial_{\beta} Q\right)
\end{aligned}
$$

$$
\begin{aligned}
& +\left(2|\beta|^{2}+1\right)\left(\bar{\alpha} \partial_{\bar{\alpha}} Q-\alpha \partial_{\alpha} Q\right) \\
& +2\left[\left(\bar{\alpha}^{2} \beta \partial_{\bar{\beta}} Q-\alpha^{2} \bar{\beta} \partial_{\beta} Q\right)\right. \\
& \left.+\left(\alpha \bar{\beta}^{2} \partial_{\bar{\alpha}} Q-\bar{\alpha} \beta^{2} \partial_{\alpha} Q\right)\right] \\
& +2\left(\bar{\alpha} \bar{\beta} \partial_{\bar{\alpha}} \partial_{\bar{\beta}} Q-\alpha \beta \partial_{\alpha} \partial_{\beta} Q\right) \\
& +\left[\left(\bar{\alpha}^{2} \partial_{\bar{\beta}}^{2} Q-\alpha^{2} \partial_{\beta}^{2} Q\right)+\left(\bar{\beta}^{2} \partial_{\bar{\alpha}}^{2} Q-\beta^{2} \partial_{\alpha}^{2} Q\right)\right] \\
& =4\left[\mathcal{J}_{x^{2}}^{(1)}+\mathcal{J}_{x^{2}}^{(2)}+\mathcal{J}_{x^{2}}^{(3)}+\mathcal{J}_{x^{2}}^{(4)}\right] .
\end{aligned}
$$

where we introduced the notation $\mathcal{J}_{x^{2}}^{(i)}$ representing the $i$ th line of Eq. (A4). The dynamics of our system is governed by the von Neumann equation,

$$
\partial_{t} \rho=-i\left[H_{x^{2}}+H_{z}, \rho\right]=i h\left[J_{z}, \rho\right]+\frac{i \gamma_{x}}{2 j}\left[J_{x}^{2}, \rho\right],
$$

which is mapped into a quantum Fokker-Planck equation for the Husimi $Q$ function of the bosonic operators introduced by the Schwinger map (A1),

$$
\partial_{t} Q(\boldsymbol{\mu})=\mathcal{U}_{x^{2}}+\mathcal{U}_{z},
$$

where $\mathcal{U}_{z}=i h \mathcal{J}_{z}$ and $\mathcal{U}_{x^{2}}=\left(i \gamma_{x} / 8 j\right) \mathcal{J}_{x^{2}}$

We are interested in the entropy rate, which in the Schwinger representation is given by

$$
\frac{d S_{Q}}{d t}=-\int d^{4} \mu \mathcal{U}(Q) \ln Q=-\left(\Lambda_{x^{2}}+\Lambda_{z}\right),
$$

where $d^{4} \mu=d^{2} \alpha d^{2} \beta$ and $\Lambda_{a}=\int d^{4} \mu \mathcal{U}_{a} \ln Q$ with $a=$ $z, x^{2}$. From Eqs. (A3) and (A4) one can compute the terms of Eq. (A5). We note that the Schwinger transformation Eq. (A1) maps a bounded set of spin operators into, what is in principle, an unbounded set of bosonic operators. However, the map introduces a restriction on the set of bosonic operators, which makes the Husimi $Q$ function and its derivatives vanish for $\alpha, \beta \rightarrow \pm \infty$ once they are restricted, i.e., nonzero only to a specific region of phase space. Using this fact along with integration by parts, we find that $\Lambda_{z}=0$ and the only terms that contribute to the entropy rate are those related to diffusion, i.e., the terms with second derivatives in $\mathcal{J}_{x^{2}} . \Lambda_{x^{2}}$ can be independently computed for each term appearing in Eq. (A4). For the first contribution we have

$$
\begin{aligned}
\Lambda_{x^{2}}^{(1)} & \propto \int d^{4} \mu\left(2|\alpha|^{2}+1\right) \ln Q\left(\bar{\beta} \partial_{\bar{\beta}} Q-\beta \partial_{\beta} Q\right) \\
& =\left[\left(2|\alpha|^{2}+1\right) \bar{\beta} Q \ln Q-\text { c.c. }\right]_{-\infty}^{\infty}-\int d^{4} \mu\left(2|\alpha|^{2}+1\right)\left(\ln Q+\bar{\beta} \frac{\partial_{\bar{\beta}} Q}{Q}-\ln Q-\beta \frac{\partial_{\beta} Q}{Q}\right) Q \\
& =-\int d^{4} \mu\left(2|\alpha|^{2}+1\right)\left(\bar{\beta} \partial_{\bar{\beta}} Q-\beta \partial_{\beta} Q\right) \\
& =-\left[\left(2|\alpha|^{2}+1\right) \bar{\beta} Q-\text { c.c. }\right]_{-\infty}^{\infty}+\int d^{4} \mu\left(2|\alpha|^{2}+1\right)(Q-Q)=0
\end{aligned}
$$

the last term in the first line of Eq. (A4) is structurally the same as the one above and hence it vanishes. The second contribution is given by

$$
\begin{aligned}
\Lambda_{x^{2}}^{(2)} & \propto \int d^{4} \mu\left(\bar{\alpha}^{2} \beta \partial_{\bar{\beta}} Q-\alpha^{2} \bar{\beta} \partial_{\beta} Q\right) \ln Q=\left[Q \ln Q \bar{\alpha}^{2} \beta-\text { c.c. }\right]_{-\infty}^{\infty}-\int d^{4} \mu \bar{\alpha}^{2} \beta \partial_{\bar{\beta}} Q-\alpha^{2} \bar{\beta} \partial_{\beta} Q \\
& =-\left[\bar{\alpha}^{2} \beta Q-\text { c.c. }\right]_{-\infty}^{\infty}+\int d^{4} \mu\left(\partial_{\bar{\beta}}\left[\bar{\alpha}^{2} \beta\right]-\partial_{\beta}\left[\alpha^{2} \beta\right]\right) Q=0,
\end{aligned}
$$


and the same result holds for the last contribution of the second line of Eq. (A4). The first nonvanishing contribution comes from the third line,

$$
\Lambda_{x^{2}}^{(3)} \propto \int d^{4} \mu \ln Q\left(\bar{\alpha} \bar{\beta} \partial_{\bar{\alpha}} \partial_{\bar{\beta}} Q-\alpha \beta \partial_{\alpha} \partial_{\beta} Q\right)=-\int d^{4} \mu\left(\bar{\alpha} \frac{\partial_{\bar{\alpha}} Q}{Q} \bar{\beta} \frac{\partial_{\bar{\beta}} Q}{Q}-\text { c.c. }\right) Q
$$

and, finally,

$$
\begin{aligned}
\Lambda_{x^{2}}^{(4)} & \propto \int d^{4} \mu \ln Q\left(\bar{\alpha}^{2} \partial_{\bar{\beta}}^{2} Q-\alpha^{2} \partial_{\beta}^{2} Q\right)=\left[\bar{\alpha} \ln Q \partial_{\bar{\beta}} Q-\text { c.c. }\right]_{-\infty}^{\infty}-\int d^{4} \mu \bar{\alpha}^{2} \frac{\left(\partial_{\bar{\beta}} Q\right)^{2}}{Q}-\text { c.c. } \\
& =-\int d^{4} \mu\left(\bar{\alpha} \frac{\partial_{\bar{\beta}} Q}{Q}\right)^{2} Q-\text { c.c. },
\end{aligned}
$$

the second term of $\mathcal{J}_{x^{2}}^{(4)}$ is structurally the same as the first, it suffices to substitute $\alpha \rightarrow \beta$ in Eq. (A9). Hence, we obtain that the nonvanishing contribution to $\Lambda_{x^{2}}$ is

$$
\Lambda_{x^{2}}=-\frac{i \gamma_{x}}{8 j} \int d^{4} \mu\left[\left(\bar{\alpha} \frac{\partial_{\bar{\beta}} Q}{Q}\right)^{2}+2 \bar{\alpha} \bar{\beta} \frac{\partial_{\bar{\alpha}} Q}{Q} \frac{\partial_{\bar{\beta}} Q}{Q}+\left(\bar{\beta} \frac{\partial_{\bar{\alpha}} Q}{Q}\right)^{2}-\text { c.c. }\right] Q=-\frac{i \gamma_{x}}{8 j} \int d^{4} \mu\left[\left(\bar{\alpha} \frac{\partial_{\bar{\beta}} Q}{Q}+\bar{\beta} \frac{\partial_{\bar{\alpha}} Q}{Q}\right)^{2}-\text { c.c. }\right] Q .
$$

The contribution due to $\Lambda_{z}$ vanishes because it has exactly the same structure as $\Lambda_{x}^{(1)}$ in Eq. (A6). Now Eq. (A10) can be written as

$$
\Lambda_{x^{2}}=\frac{\gamma_{x}}{4 j} \Im\left\langle\left(\bar{\alpha} \frac{\partial_{\bar{\beta}} Q}{Q}+\bar{\beta} \frac{\partial_{\bar{\alpha}} Q}{Q}\right)^{2}\right\rangle=-\frac{\gamma_{x}}{4 j} \Im\left\langle\left(\alpha \frac{\partial_{\beta} Q}{Q}+\beta \frac{\partial_{\alpha} Q}{Q}\right)^{2}\right\rangle
$$

thus leading us to the following expression for the entropy rate:

$$
\frac{d S_{Q}}{d t}=-\Lambda_{x^{2}}=\frac{\gamma_{x}}{4 j} \Im\left\langle\left(\alpha \frac{\partial_{\beta} Q}{Q}+\beta \frac{\partial_{\alpha} Q}{Q}\right)^{2}\right\rangle
$$

In this equation, $\langle\ldots\rangle$ means an average over the phase space.

For a fixed the value of $j$, we can go back to the polar representation $(\theta, \phi)$ using the following relations [48]:

$$
\begin{gathered}
\alpha \partial_{\beta}=e^{-i \phi} \cos ^{2} \frac{\theta}{2}\left[2 j \tan \frac{\theta}{2}+\partial_{\theta}-i \frac{\partial_{\phi}}{\sin \theta}\right], \\
\beta \partial_{\alpha}=e^{i \phi} \sin ^{2} \frac{\theta}{2}\left[2 j \cot \frac{\theta}{2}-\partial_{\theta}+i \frac{\partial_{\phi}}{\sin \theta}\right],
\end{gathered}
$$

since $S_{Q}(\mu)=-\int d^{4} \mu \ln Q(\mu) Q(\mu) \rightarrow S_{Q}(\Omega)=-(2 j+1) / 4 \pi \int d \Omega \ln Q(\Omega) Q(\Omega)$ which gives us the following expression for the entropy production in polar coordinates:

$$
\frac{d S_{\Omega}}{d t}=\frac{2 j+1}{16 \pi j} \gamma_{x} \Im \int d \Omega \frac{1}{Q(\Omega)}\left(e^{-i \phi} \cos ^{2} \frac{\theta}{2}\left[2 j \tan \frac{\theta}{2}+\partial_{\theta}-i \frac{\partial_{\phi}}{\sin \theta}\right] Q(\Omega)+e^{i \phi} \sin ^{2} \frac{\theta}{2}\left[2 j \cot \frac{\theta}{2}-\partial_{\theta}+i \frac{\partial_{\phi}}{\sin \theta}\right] Q(\Omega)\right)^{2} .
$$

\section{APPENDIX B: NUMERICAL STUDIES ON THE LMG MODEL}

In studying the DPT of the LMG model, care must be taken with the fact that the ground state is twofold degenerate. We therefore define an echo for each ground state, $\mathcal{L}_{\alpha}=$ $\left|\left\langle\psi_{0}^{\alpha} \mid \psi_{t}^{\alpha}\right\rangle\right|^{2}$, and consider only the net rate,

$$
r_{s}(t)=-\frac{1}{N} \log \left[\sum_{\alpha=1}^{g} \mathcal{L}_{\alpha}\right]
$$

which therefore captures the total return probability. However, as shown [10], in the thermodynamic limit this converges to the minimum among all the contributions,

$$
r_{m} \equiv \lim _{N \rightarrow \infty} r(t)=-\frac{1}{N} \log \left[\min _{\alpha} \mathcal{L}_{\alpha}\right]
$$

It is for this reason that in the main text it sufficed to consider only the rate starting from a single ground state.

In this Appendix we describe several numerical studies of the LMG model. Specifically, we consider four distinct quench processes, from $h_{0}=0$ to $h=0.1,0.8,1.0$, and 1.6. Note that the first two quenches occur before the quantum critical point while the last one occurs after. 

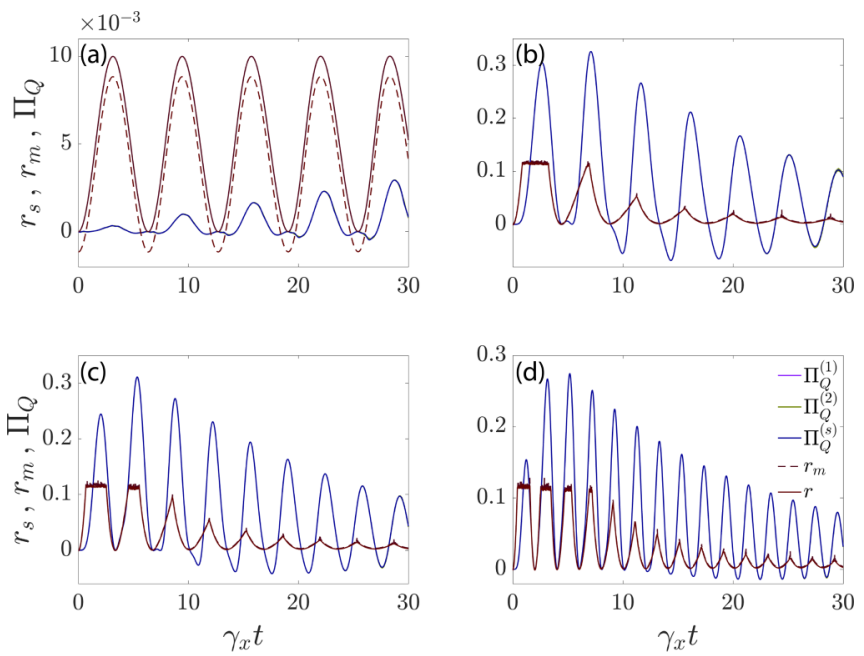

FIG. 13. Comparison between $r$ and $r_{m}$. From top to bottom and from left to right we consider quenches from $h_{0}=0$ to $h=0.1,0.8$, 1.0 , and 1.6

\section{The dynamical quantum phase transition}

We start by showing the Loschmidt echo for the two ground states of the system in Figs. 6 and 7. As we can see from these figures, the behavior of $\mathcal{L}$ regarding the dynamical quantum phase transition is independent of the considered initial state. However, it does depend on the quench amplitude and show no sensible difference at the quantum critical point $h=1$.

To make this point more clear investigate the rate functions $r$ and $r_{m}$, depicted in Fig. 8. We can see that $r_{s}$ converges to $r_{m}$ when we approach the thermodynamic limit.

\section{Entropy production}

Figures 9 and 10 show the dynamical behavior of the entropy for the two ground states. We can observe the same qualitative behavior for all the considered quenches, except for the smaller one. The entropy approaches a maximum as we approach the thermodynamic limit. The oscillation pattern presented in these plots signal the dynamical quantum phase transitions.

In order to show this fact, we consider the entropy production rate, given by the time derivative of $S_{Q}$. This is shown in
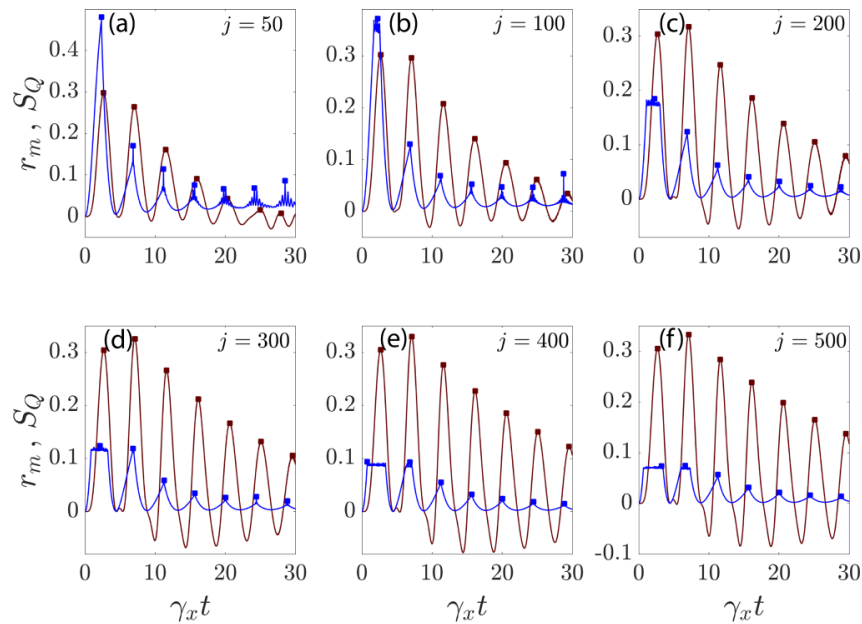

FIG. 14. Comparison between the maximums of the entropy production rate and the nonanalytical points of the rate function for the quench $h_{0}=0$ to $h=0.8$.

Figs. 11 and 12 for the same states addressed in the case of the entropy. Again, except for the small quench, the qualitative behavior of this quantity presents several maximums and we show in the main text and in the next subsection that such maximums are related to the dynamical quantum phase transitions.

\section{Entropy production rate and the rate function}

Finally, we address here the main message of the present article. Figure 13 shows the comparison between the entropy production rate and the rate function $r_{m}$ for a fixed value of the angular momentum $(j=500)$. Except for the case of small quench, where we actually do not have a quantum phase transition since $r_{m}$ is analytical in time, the other considered quenches clearly shows that the entropy production rate is able to sign the dynamical quantum phase transition, as discussed in the main text.

This relation can be deeply stated if we consider the times at which entropy production rate shows a maximum and the critical times, where we have a dynamical quantum phase transition. This is done in Fig. 14, where a clear linear behavior emerges, thus supporting our claims in the main text. The points highlighted in this figure are the ones shown in Fig. 4 of the main text.
[1] M. Heyl, A. Polkovnikov, and S. Kehrein, Dynamical Quantum Phase Transitions in the Transverse-Field Ising Model, Phys. Rev. Lett. 110, 135704 (2013).

[2] S. Vajna and B. Dóra, Disentangling dynamical phase transitions from equilibrium phase transitions, Phys. Rev. B 89, 161105(R) (2014).

[3] J. C. Halimeh and V. Zauner-Stauber, Dynamical phase diagram of quantum spin chains with long-range interactions, Phys. Rev. B 96, 134427 (2017).
[4] C. Karrasch and D. Schuricht, Dynamical phase transitions after quenches in nonintegrable models, Phys. Rev. B 87, 195104 (2013).

[5] E. Canovi, P. Werner, and M. Eckstein, First-Order Dynamical Phase Transitions, Phys. Rev. Lett. 113, 265702 (2014).

[6] M. Heyl, Quenching a quantum critical state by the order parameter: Dynamical quantum phase transitions and quantum speed limits, Phys. Rev. B 95, 060504(R) (2017). 
[7] P. Jurcevic, H. Shen, P. Hauke, C. Maier, T. Brydges, C. Hempel, B. P. Lanyon, M. Heyl, R. Blatt, and C. F. Roos, Direct Observation of Dynamical Quantum Phase Transitionsin an Interacting Many-Body System, Phys. Rev. Lett. 119, 080501 (2017).

[8] N. Fläschner, D. Vogel, M. Tarnowski, B. S. Rem, D.-S. Lühmann, M. Heyl, J. C. Budich, L. Mathey, K. Sengstock, and C. Weitenberg, Observation of dynamical vortices after quenches in a system with topology, Nat. Phys. 14, 265 (2018).

[9] J. Zhang, G. Pegano, P. W. Hess, A. Kyprianidis, P. Becker, H. Kaplan, A. V. Gorshkov, Z.-X. Gong, and C. Monroe, Observation of a many-body dynamical phase transition with a 53-qubit quantum simulator, Nature 551, 601 (2017).

[10] M. Heyl, Dynamical quantum phase transitions: A review, Rep. Prog. Phys. 81, 054001 (2018).

[11] M. Heyl, Dynamical quantum phase transition: A brief survey, Europhys. Lett. 125, 26001 (2019).

[12] A. A. Zvyagina, Dynamical quantum phase transitions, Low Temp. Phys. 42, 971 (2016).

[13] C. Yang and T. Lee, Statistical theory of equations of state and phase transitions. I. Theory of condensation, Phys. Rev. 87, 404 (1952).

[14] M. E. Fisher, in Boulder Lectures in Theoretical Physics (University of Colorado, Boulder, 1965), Vol. 7.

[15] M. Campisi and J. Goold, Thermodynamics of quantum information scrambling, Phys. Rev. E 95, 062127 (2017).

[16] A. Altland and F. Haake, Quantum Chaos and Effective Thermalization, Phys. Rev. Lett. 108, 073601 (2012).

[17] A. Wehrl, General properties of entropy, Rev. Mod. Phys. 50, 221 (1978).

[18] A. Wehrl, On the relation between classical and quantum-mechanical entropy, Rep. Math. Phys. 16, 353 (1979).

[19] V. Buzek, C. H. Keitel, and P. L. Knight, Sampling entropies and operational phase-space measurement. I. General formalism, Phys. Rev. A 51, 2575 (1995).

[20] H. Lipkin, N. Meshkov, and A. Glick, Validity of manybody approximation methods for a solvable model: (I), Exact solutions and perturbation theory, Nucl. Phys. 62, 188 (1965).

[21] N. Meshkov, A. Glick, and H. Lipkin, Validity of many-body approximation methods for a solvable model: (II). Linearization procedures, Nucl. Phys. 62, 199 (1965).

[22] A. Glick, H. Lipkin, and N. Meshkov, Validity of many-body approximation methods for a solvable model: (III). Diagram summations, Nucl. Phys. 62, 211 (1965).

[23] J. I. Cirac, M. Lewenstein, K. Mølmer, and P. Zoller, Quantum superposition states of Bose-Einstein condensates, Phys. Rev. A 57, 1208 (1998).

[24] D. A. Garanin, X. Martínez Hidalgo, and E. M. Chudnovsky, Quantum-classical transition of the escape rate of a uniaxial spin system in an arbitrarily directed field, Phys. Rev. B 57, 13639 (1998).

[25] J. I. Latorre, R. Orús, E. Rico, and J. Vidal, Entanglement entropy in the Lipkin-Meshkov-Glick model, Phys. Rev. A 71, 064101 (2005).
[26] J. Vidal, G. Palacios, and R. Mosseri, Entanglement in a secondorder quantum phase transition, Phys. Rev. A 69, 022107 (2004).

[27] J. Vidal, R. Mosseri, and J. Dukelsky, Entanglement in a first-order quantum phase transition, Phys. Rev. A 69, 054101 (2004).

[28] P. Ribeiro, J. Vidal, and R. Mosseri, Thermodynamical Limit of the Lipkin-Meshkov-Glick Model, Phys. Rev. Lett. 99, 050402 (2007).

[29] P. Ribeiro, J. Vidal, and R. Mosseri, Exact spectrum of the Lipkin-Meshkov-Glick model in the thermodynamic limit and finite-size corrections, Phys. Rev. E 78, 021106 (2008).

[30] P. Ribeiro and T. Paul, Semiclassical analysis of spin systems near critical energies, Phys. Rev. A 79, 032107 (2009).

[31] S. Dusuel and J. Vidal, Finite-Size Scaling Exponents of the Lipkin-Meshkov-Glick Model, Phys. Rev. Lett. 93, 237204 (2004).

[32] S. Dusuel and J. Vidal, Continuous unitary transformations and finite-size scaling exponents in the Lipkin-Meshkov-Glick model, Phys. Rev. B 71, 224420 (2005).

[33] A. Das, K. Sengupta, D. Sen, and B. K. Chakrabarti, Infiniterange Ising ferromagnet in a time-dependent transverse magnetic field: Quench and ac dynamics near the quantum critical point, Phys. Rev. B 74, 144423 (2006).

[34] Y. Hamdouni and F. Petruccione, Time evolution and decoherence of a spin- $1 / 2$ particle coupled to a spin bath in thermal equilibrium, Phys. Rev. B 76, 174306 (2007).

[35] J. Bao, B. Guo, H.-G. Cheng, M. Zhou, Jin Fu, Y.-C. Deng, and Z.-Y. Sun, Multipartite nonlocality in the Lipkin-MeshkovGlick model, Phys. Rev. A 101, 012110 (2020).

[36] B. Žunkovič, M. Heyl, M. Knap, and A. Silva, Dynamical Quantum Phase Transitions in Spin Chains with Long-Range Interactions: Merging different Concepts of Nonequilibrium Criticality, Phys. Rev. Lett. 120, 130601 (2018).

[37] J. Lang, B. Frank, and J. C. Halimeh, Concurrence of dynamical phase transitions at finite temperature in the fully connected transverse-field Ising model, Phys. Rev. B 97, 174401 (2018).

[38] I. Homrighausen, N. O. Abeling, V. Zauner-Stauber, and J. C. Halimeh, Anomalous dynamical phase in quantum spin chains with long-range interactions, Phys. Rev. B 96, 104436 (2017).

[39] J. Lang, B. Frank, and J. C. Halimeh, Dynamical Quantum Phase Transitions: A Geometric Picture, Phys. Rev. Lett. 121, 130603 (2018).

[40] O. Castaños, R. López-Peña, J. G. Hirsch, and E. LópezMoreno, Classical and quantum phase transitions in the Lipkin-Meshkov-Glick model, Phys. Rev. B 74, 104118 (2006).

[41] K. Takahashi and N. Saitôn, Chaos and Husimi Distribution Function in Quantum Mechanics, Phys. Rev. Lett. 55, 645 (1985).

[42] F. Mintert and K. Życzkowski, Wehrl entropy, Lieb conjecture, and entanglement monotones, Phys. Rev. A 69, 022317 (2004).

[43] G. Palma, Uncertainty relations with quantum memory for the Wehrl entropy, Lett. Math. Phys. 108, 2139 (2018).

[44] O. Castaños, M. Calixto, F. Pérez-Bernal, and E. Romera, Identifying the order of a quantum phase transition by means of Wehrl entropy in phase space, Phys. Rev. E 92, 052106 (2015). 
[45] J. P. Santos, L. C. Céleri, F. Brito, G. T. Landi, and M. Paternostro, Spin-phase-space-entropy production, Phys. Rev. A 97, 052123 (2018).

[46] B. O. Goes, C. E. Fiore, and G. T. Landi, Quantum features of entropy production in driven-dissipative transitions, Phys. Rev. Res. 2, 013136 (2020).
[47] T. Holstein and H. Primakoff, Field dependence of the intrinsic domain magnetization of a ferromagnet, Phys. Rev. 58, 1098 (1940).

[48] Y. Takahashi and F. Shibata, Spin coherent state representation in non-equilibrium statistical mechanics, J. Phys. Soc. Jpn. 38, 656 (1975). 